Publication to be submitted:

Proceedings of the IET - Circuits, Devices and Systems

Reference:

Revision of CDS-2007-0320

\title{
Title: \\ A Compact CMOS Current Conveyor for Integrated NEMS Resonators
}

\author{
Authors: \\ Julien Arcamone ${ }^{1}$, Bertrand Misischi ${ }^{1}$, Francisco Serra-Graells ${ }^{1}$, \\ Marc A.F. van den Boogaart ${ }^{2}$, Jürgen Brugger ${ }^{2}$, Francesc Torres ${ }^{3}$, \\ Gabriel Abadal ${ }^{3}$, Núria Barniol ${ }^{3}$ and Francesc Pérez-Murano ${ }^{1}$ \\ ${ }^{1}$ Institut de Microelectrònica de Barcelona, \\ Centro Nacional de Microelectrónica - CSIC \\ ${ }^{2}$ Microsystems Laboratory, Ecole Polytechnique Fédérale de Lausanne (EPFL) \\ ${ }^{3}$ Departament d'Enginyeria Electrònica, Universitat Autònoma de Barcelona
}

Correspondence Author:

Francisco Serra-Graells

mailto: paco.serra@cnm.es

http://www.cnm.es/ pserra

Tel: +34935947700

Fax: +34935801496

Institut de Microelectrònica de Barcelona

Centro Nacional de Microelectrónica - CSIC

Campus UAB

08193 Bellaterra

Spain

Statistics:

Number of words: $\sim 2140$

Number of figures: 10

Number of tables: 4

Number of references: 16 


\title{
A Compact CMOS Current Conveyor for Integrated NEMS Resonators
}

\author{
Julien Arcamone ${ }^{1}$, Bertrand Misischi ${ }^{1}$, Francisco Serra-Graells ${ }^{1}$, \\ Marc A.F. van den Boogaart ${ }^{2}$, Jürgen Brugger ${ }^{2}$, Francesc Torres ${ }^{3}$, \\ Gabriel Abadal ${ }^{3}$, Núria Barniol ${ }^{3}$ and Francesc Pérez-Murano ${ }^{1}$ \\ ${ }^{1}$ Institut de Microelectrònica de Barcelona, Centro Nacional de Microelectrónica - CSIC \\ ${ }^{2}$ Microsystems Laboratory, Ecole Polytechnique Fédérale de Lausanne (EPFL) \\ ${ }^{3}$ Departament d'Enginyeria Electrònica, Universitat Autònoma de Barcelona
}

January 15, 2008

\begin{abstract}
This paper presents a fully-integrated NEMS resonator together with a compact built-in CMOS interfacing circuitry. The proposed low-power CCII circuit allows measuring the mechanical frequency response of the nanocantilever structure in the $\mathrm{MHz}$ range. Detailed experimental results at different DC biasing conditions and pressure levels are presented for a real mixed electro-mechanical system integrated through a combination of in-house standard CMOS technology and nanodevice postprocessing based on nanostencil lithography. The proposed read out circuit can be adapted to operate the nanocantilever in closed loop as a stand alone oscillator.
\end{abstract}

\section{Introduction}

Recently, nanotechnology has become a promising approach to integrate both sensors $[1,2]$ and actuators $[3,4]$ in CMOS technologies. Also, electromechanical devices can save power consumption and silicon area for specific functions compared to their purely electronic counterparts, specially in mobile applications. In particular, the possibility of using nanoelectromechanical systems (NEMS) to replace the costly and bulky quartz crystal devices is of high interest for the implementation of integrated oscillators [5,6].

However, in order to take advantage of the emerging nanotechnologies, the resulting nanodevices must be compatible with standard CMOS processing, and specific circuits 
have to be developed either for testing these NEMS or simply for their interfacing within the final mixed electromechanical system-on-chip.

This paper presents both, a fully-integrated nanocantilever operable at frequencies in the $\mathrm{MHz}$ range, together with a specific low-power and compact CMOS read out circuit for its experimental characterization and interfacing. From a technological point of view, the NEMS fabrication is based on nanostencil lithography [7], while from the design side a new low-power CMOS interface topology is proposed based on second generation current conveyors (CCII) [8]. Finally, the mixed electro-mechanical system is fully integrated through a combination of in-house standard CMOS technology and nanodevice post-processing [9].

Next section introduces a general overview of the mixed NEMS-CMOS system. Then, the modeling of the nanoresonator device is explained in Section 3, while the novel lowpower CCII CMOS topology for its interface is proposed in Section 4. Experimental results of the resulting mixed integrated circuit are reported in Section 5, and conclusions are finally summarized in Section 6.

\section{NEMS Read Out Scheme}

Schematically, NEMS resonators based on nanocantilevers include the main parts shown in Figure 1(a). The device consists of a driver, mechanically anchored, and a cantilever placed very close to the driver and clamped at one end only, so it can freely bend around the static position at a given oscillation frequency.

[Figure 1 about here.]

Following the ideal read out scheme illustrated in the same Figure 1(a), the fixed driver is used to bias the required DC voltage $V_{\text {static }}$ (typically between $1 \mathrm{~V}$ and $20 \mathrm{~V}$ ) and to act as the input terminal for the frequency stimulation $V_{\text {osc }}$ (typically from $-30 \mathrm{dBm}$ to $0 \mathrm{dBm}$ ). On the other hand, the cantilever acts as the output terminal, allowing the read out of the NEMS resonator output current $I_{\text {res }}$ (typically in the $\mathrm{nA}$ range) and the corresponding voltage signal $V_{\text {meas }}$ across the load resistor $R_{\text {load }}$. The resonance frequency $f_{\text {res }}$ (typically from $1 \mathrm{MHz}$ to $10 \mathrm{MHz}$ ) depends on the cantilever material and dimensions, as detailed later on in Section 3.

Unfortunately, the ideal read out scheme of Figure 1(a) is not feasible in practice due to the $\mathrm{M} \Omega$ range values of the NEMS resonator at $f_{\text {res }}$, which limits the allowed output capacitance $C_{\text {load }}$ far below the $\mathrm{pF}$ range. Hence, a built-in interface circuit is 
required. Several implementations based on the passive integration of $I_{\text {res }}$ through $C_{\text {par }}$ have been reported in the literature $[6,10,11]$. However, the resulting integration gain is still strongly dependent on the layout parasitics. In order to solve this drawback, the alternative strategy of Figure 1(b) is proposed, where a built-in CMOS CCII is inserted in between the NEMS resonator and the output. This new scheme is equivalent to Figure 1(a) for $V_{\text {static }}=V_{\text {bias }}-V_{\text {ref }}$, but it addresses the previous drawback in two directions: the new resonator output capacitance $C_{\text {par }}$ can be easily kept in the sub-pF range as it is no more related with $C_{l o a d}$, and also its effects are minimized by ensuring a constant voltage bias at the output of the resonator. Furthermore, the proposed CCII interface amplifies $I_{\text {res }}$ for either external measurement at $V_{\text {meas }}$ or internal feedback to $V_{\text {osc }}$ (e.g. stand alone oscillator), as detailed in Section 4.

\section{NEMS Resonator}

The basis of the fabrication process is described in [10] and consists of post-processing standard CMOS wafers, where a polysilicon area for nanodevice integration has been reserved. However, the novelty here is the lithography technique employed for patterning the nanodevices: an enhanced resolution down to $200 \mathrm{~nm}$ and full-wafer parallel processing are obtained [9] by applying nanostencil lithography (nSL) [7]. In this new process, and after concluding the fabrication of the CMOS circuits, nanodevice areas are selectively patterned with a $80 \mathrm{~nm}$ thick aluminum layer by nSL. Subsequent process steps consist on reactive ion etching of silicon to transfer the aluminum pattern to the polysilicon structural layer, wafer dicing and silicon oxide wet etching to release the mechanical structure, combined with a critical point drying process $\left(\mathrm{CO}_{2}\right.$ dryer $)$ in order to avoid stiction phenomena. Following this procedure, surrounding CMOS circuits show no degradation of their analog performance.

As a result of the above CMOS post-processing, the polysilicon structure of Figure 2(a) is obtained, where $W, L, H$ and $D$ stand for the cantilever width, length, height and gap to driver, respectively. In our case, typical dimensions for the NEMS device are listed in Table 1.

[Figure 2 about here.]

[Table 1 about here.] 
According to $[12,13]$, the use of continuum mechanics is still valid to predict the mechanical behavior of resonating structures with cross-sectional areas larger than few $\mathrm{nm}^{2}$. As a result, the analytical expression of the natural (i.e. without any electrostatic force) resonance frequency for undamped (i.e. ideal vacuum) lateral flexion is found to be:

$$
f_{r e s}=\frac{1.015}{2 \pi} \sqrt{\frac{E}{\rho}} \frac{W}{L^{2}}
$$

where $E$ and $\rho$ stand for the Young's modulus and the density of the nanocantilever material, respectively. Around the resonance frequency of the considered mode, the nanocantilever moves laterally and its mechanical motion is translated into an electrical signal. In fact, the read out is based on capacitive detection according to:

$$
\begin{aligned}
I_{\text {res }} & =\frac{d Q_{\text {res }}}{d t} \\
& =\left(C_{\text {stat }}+C_{\text {mot }}\right) \frac{d V_{\text {osc }}}{d t}+\left(V_{\text {bias }}-V_{\text {ref }}+V_{\text {osc }}\right) \frac{d C_{\text {mot }}}{d t} \\
& \simeq C_{\text {stat }} \frac{d V_{\text {osc }}}{d t}+\left(V_{\text {bias }}-V_{\text {ref }}\right) \frac{d C_{\text {mot }}}{d t}
\end{aligned}
$$

where $C_{\text {stat }}$ and $C_{m o t}$ are the static plate and the motion capacitances, respectively. Thus, the NEMS output current is a sum of two contributions: one arising from the static structure (i.e. $C_{s t a t} \frac{d V_{o s c}}{d t}$ ) and the other coming from the nanocantilever motion itself (i.e. $\left.\left(V_{\text {bias }}-V_{\text {ref }}\right) \frac{d C_{m o t}}{d t}\right)$. This second part allows the measure of the frequency response of the mechanical resonator.

In practice, either due to air environment or other second order effects, the NEMS resonator can exhibit important losses that translate into a decrease of its quality factor $Q$. In these cases, the nanomechanical resonator can be described through a small signal equivalent RLC model [5], as depicted in Figure 2(b). The main physical parameters of this model are the cantilever dissipation, mass and elasticity, which are electrically equivalent to $R_{\text {res }}, L_{r e s}$ and $C_{r e s}$ respectively, and the static plate capacitance $C_{\text {stat }}$. In addition, the cantilever output capacitance $C_{\text {cant }}$ and the fringing coupling to the driver $C_{\text {coup }}$ are included here, while the driver own capacitance $C_{d r i v}$ can be neglected according to the read out scheme of Figure 1(b). It is important to note that $R_{r e s}$ depends on the $Q$ factor, whose theoretical calculation is difficult to obtain. Therefore, $R_{\text {res }}$ is estimated from experimental data of $Q$, as illustrated in Section 5 . 


\section{Current Conveyor Circuit}

As already argued, the purpose of the CMOS interfacing circuit in Figure 1 is to ensure a constant bias at the output of the NEMS resonator and to read out its capacitive current. In this sense, the compact CMOS circuit shown in Figure 3 is proposed, which consists of an input low-impedance stage (M1-M4) and an output current scaler (M5-M12).

[Figure 3 about here.]

Firstly, the input low-impedance is achieved by the cascode transistor M4, which is continuously controlled by the telescopic differential amplifier M1-M3. According to the advanced EKV MOSFET model [14], the resulting small-signal input resistance at $X$ is found to be:

$$
r_{i n}=\left(\frac{1}{n+\frac{g m_{g 1}}{g m_{d 1}}}\right) \frac{1}{g m_{g 4}}
$$

where $n$ stands for the subthreshold slope factor. Hence, the error amplifier M1 scales

down $r_{i n}$ by the gain factor $\frac{g m_{g 1}}{g m_{d 1}}$ compared to the impedance of the single M4 transistor $\frac{1}{g m_{g 4}}$. As a result, this stage generates an input voltage $V_{X}$ that follows $V_{Y}$.

Secondly, the NEMS current sensed by M4 is amplified by the geometry scaling factors $M=\frac{(W / L)_{7,8}}{(W / L)_{5,6}}$ and $N=\frac{(W / L)_{11,12}}{(W / L)_{9,10}}$ of the two-stage cascode current mirrors M5-M8 and M9-M12 biased at $V_{\text {casp }}$ and $V_{\text {casn }}$, respectively. In order to reduce the overall power consumption, a $K / M$ fraction of the biasing is subtracted before the second amplification stage.

In conclusion, the proposed circuit qualitatively behaves like a classic CCII- [8], but with an extra gain from the $I_{X}$ to $I_{Z}$ signals:

$$
\left[\begin{array}{c}
I_{Y} \\
V_{X} \\
I_{Z}
\end{array}\right]=\left[\begin{array}{ccc}
0 & 0 & 0 \\
1 & 0 & 0 \\
0 & -M N & 0
\end{array}\right]\left[\begin{array}{c}
V_{Y} \\
I_{X} \\
V_{Z}
\end{array}\right]
$$

In fact, the new CCII- topology introduced in Figure 3 is an improvement of the input stage [15] in order to allow a wider voltage range for both $V_{X}$ and $V_{Y}$ thanks to the symmetry of the M1 and M2 drain connections. Also, compared to other similar CCII- evolutions like [16], the proposed circuit saves power consumption by minimizing the transistor count of the input stage. 
Applying the above circuit model to the general read out scheme of Figure 1, we obtain the final design equations:

$$
\Delta V_{\text {meas }}=R_{\text {load }} M N \Delta I_{\text {res }} \quad \text { and } \quad V_{\text {cant }} \equiv V_{\text {ref }}
$$

where $V_{\text {cant }}$ stands for the voltage biasing at the NEMS resonator output. The MOS device dimensions for the proposed CCII- are listed in Table 2, while the simulated performance is summarized in Table 3 and illustrated in Figure 4 for a typical set of design values. Thanks to the low transistor count of the proposed CCII-, the CMOS interfacing circuit is compatible with low-power operation and compact integration.

[Table 2 about here.]

[Table 3 about here.]

[Figure 4 about here.]

\section{Experimental Results}

Following the proposals of Sections 3 and 4, a compact 1.5MHz NEMS resonator together with the CMOS interfacing circuit has been integrated through the in-house standard CMOS double polysilicon technology and the full-wafer post-processing steps based on nanostencil lithography described in [9]. As shown in Figure 5, the resulting size of the complete mixed electromechanical circuit without pads is around $800 \mu \mathrm{m} \times 400 \mu \mathrm{m}\left(0.32 \mathrm{~mm}^{2}\right)$. Since the in-house CMOS technology is a $2.5 \mu \mathrm{m}$ lithography process, a considerably smaller implementation can be obtained using modern submicron CMOS technologies.

[Figure 5 about here.]

Taking advantage of the built-in interfacing circuit, the NEMS resonator has been characterized. In this sense, the typical transfer function of the nanoresonator measured in vacuum is depicted in Figure 6. As it can be easily seen, the NEMS device exhibits a clear and narrow mechanical resonance around $f_{r e s}=1.5 \mathrm{MHz}$, which matches with theoretical estimations within $\pm 5 \%$, showing important magnitude losses outside this band. The mechanical $Q$ factor is estimated around 8500 in these vacuum conditions. All magnitude transfer functions in this section are normalized to $R_{\text {load }} M N / R_{\text {res }}$. However, in case of fully integrated closed loop operation (e.g. stand alone oscillator), this attenuation factor 
can be compensated up to $0 \mathrm{~dB}$ by choosing larger $M, N$ and $R_{\text {load }}$ design values, as the CCII- load capacitance is then $C_{d r i v} \ll C_{\text {load }}$.

From the $Q$ factor and the $V_{\text {bias }}-V_{\text {ref }}$ DC biasing of Figure 6, the model parameters of Table 4 are deduced. The validity of the circuit model described in Section 3 has been tested for different cantilever widths, thicknesses and cantilever/driver gaps. Apart from extracting the equivalent RLC parameters, the interfacing CMOS circuit also allowed the experimental study of the nanoresonator under different pressure and biasing conditions.

[Figure 6 about here.]

[Table 4 about here.]

In the first case, different environment pressure levels were applied in Figure 7, returning very good quality factors at vacuum levels below $10 \mathrm{~Pa}$, as illustrated in Figure 8 . The most pronounced evolution occurs above $10 \mathrm{~Pa}$, where the $Q$ factor can be enhanced by three orders of magnitude, while it tends to saturate not far below 10Pa. These results confirm that viscous damping plays a key role regarding the value of the quality factor of nano and microresonators, whereas at low pressure intrinsic loss mechanisms dominate.

[Figure 7 about here.]

[Figure 8 about here.]

Finally, different biasing levels has been tested in Figure 9 using the same circuit. These results fit the dependence of the resonance frequency on the square of the DC biasing, as depicted in Figure 10. The negative slope here corresponds to a classical spring-softening case, which means that the electrostatic effect dominates over mechanical stiffening when applying an electrostatic driving force.

[Figure 9 about here.]

[Figure 10 about here.]

\section{Conclusions}

A compact NEMS resonator together with its CMOS interfacing circuitry has been successfully integrated and experimentally characterized. The new low-power CCII read out circuit allows detailed measurements in the $\mathrm{MHz}$ range of the nanodevice for model extraction 
under different pressure and DC biasing conditions. In this sense, the proposed interfacing circuit can be easily adapted (e.g. choosing a larger value for $R_{\text {load }}$ and scaling factors) to allow the close loop operation of the nanocantilever as a mixed electro-mechanical stand alone oscillator.

\section{Acknowledgments}

This work has been partially funded by the European Union (NaPa:NMP4-CT-2003500120) and the Spanish government (NanoSys:TIC2003-07237).

\section{References}

[1] A. V. Schaik and S. Shamma. A Neuromorphic Sound Localizer for a Smart MEMS System. Journal of Analog Integrated Circuits and Signal Processing, Kluwer Academic Publishers, 39:267-273, 2004.

[2] H. P. Le, K. Shah, J. Singh, and A. Zayegh. Design and Implementation of An Omptimised Wireless Pressure Sensor for Biomedical Application. Journal of Analog Integrated Circuits and Signal Processing, Springer Engineering, 48:21-31, 2006.

[3] M. Tang, A. Q. Liu, A. Agarwal, Q. X. Zhang, and P. Win. A New Approach of Lateral RF MEMS Switch. Journal of Analog Integrated Circuits and Signal Processing, Kluwer Academic Publishers, 40:165-173, 2004.

[4] J.-F. Saheb, J.-F. Richard, M. Sawan, R. Meingan, and Y. Savaria. System Integration of High Voltage Electrostatic MEMS Actuators. Journal of Analog Integrated Circuits and Signal Processing, Springer Engineering, 2006. Published on-line.

[5] C. T. C. Nguyen and R. T. Howe. An Integrated CMOS Micromechanical Resonator High-Q Oscillator. IEEE Journal of Solid State Circuits, 34(4):440-455, Apr 1999.

[6] J. Verd, A. Uranga, J. Teva, J. L. López, F. Torres, J. Esteve, G. Abadal, F. PérezMurano, and N. Barniol. Integrated CMOS-MEMS with On-Chip Readout Electronics for High-Frequency Applications. IEEE Electron Device Letters, 27(6):495-497, Jun 2006.

[7] M. A. F. van den Boogart, G. M. Kim, R. Pellens, J. P. den Heuvel, and J. Brugger. Deep-Ultraviolet-Microelectromechanical Systems Stencils for High-Throughput Resistless Patterning of Mesoscopic Structures. Journal of Vacuum Science Technology B, 22(6):3174-3177, Jun 2004.

[8] A. S. Sedra and K. C. Smith. A Second-Generation Current Conveyor and Its Applications. IEEE Transactions on Circuit Theory, 17:132-134, Feb 1970.

[9] J. Arcamone, M. A. F. van den Boogaart, F. Serra-Graells, S. Hansen, J. Brugger, F. Torres, G. Abadal, N. Barniol, and F. Pérez-Murano. Full Wafer Integration of NEMS on CMOS by Nanostencil Lithography. In Proceedings of the IEEE International Electron Devices Meeting, pages 525-528, Dec 2006. 
[10] J. Verd, G. Abadal, J. Teva, M. V. Gaudo, A. Uranga, X. Borrisé, F. Campabada, J. Esteve, E. F. Costa, F. Pérez-Murano, Z. J. Davis, E. Forsen, A. Boisen, and N. Barniol. Design, Fabrication, and Characterization of a Submicroelectromechanical Resonator with Monolithically Integrated CMOS Readout Circuit. IEEE Journal of Microelectromechanical Systems, 14(3):508-519, Jun 2005.

[11] J. Verd, G. Abadal, J. Teva, A. Uranga, N. Barniol, J. Esteve, M. Duch, and F. PérezMurano. High-sensitivity Capacitive Readout System for Resonant SubmicrometerScale Cantilevers Based Sensors. In Proceedings of the International Symposium on Circuits and Systems, volume 5, pages 4209-4212, May 2005.

[12] K. L. Ekinci and M. L. Roukes. Nanoelectromechanical Systems. Review of Scientific Instruments, 76(6), 2005.

[13] Z. Tang, Y. Xu, G. Li, and N. R. Aluru. Physical Models for Coupled Electromechanical Analysis of Silicon Nanoelectromechanical Systems. Journal of Applied Physics, 97(11), 2005.

[14] C. C. Enz, F. Krummenacher, and E. A. Vittoz. An Analytical MOS Transistor Model Valid in All Regions of Operation and Dedicated to Low-Voltage and LowCurrent Applications. Journal of Analog Integrated Circuits and Signal Processing, Kluwer Academic Publishers, 8(1):83-114, 1995.

[15] W. Surakampontorn, V. Riewruja, K. Kumwachara, and K. Dejhan. Accurate CMOSbased Current Conveyors. IEEE Transactions on Instrumentation and Measurement, 40(4):699-702, Aug 1991.

[16] W. S. Hassanein, I. A. Awad, and A. M. Soliman. New Wide Band Low-Power CMOS Current Conveyors. Journal of Analog Integrated Circuits and Signal Processing, Kluwer Academic Publishers, 40:91-97, 2004. 
keywords: Low-power, current-conveyor, NEMS, resonator, read out. 


\section{List of Figures}

1 NEMS parts and ideal read out scheme (a), and proposed monolithic CMOS solution (b) . . . . . . . . . . . . . . . . . . . 13

2 Mechanical (a) and electrical equivalent circuit model (b) of the NEMS resonator. Top drawing not in scale. . . . . . . . . . . . . . . . . 14

3 Simplified schematic of the CMOS CCII- circuit. . . . . . . . . . . . . 15

4 Simulated transfer function of the CCII- circuit of Figure 3 according to the design variables of Table 3 and for different load capacitance conditions. . . 16

5 Microscope photography (top) of the mixed NEMS-CMOS circuit and SEM image (bottom) of the NEMS device. . . . . . . . . . . . . . . 17

6 Experimental NEMS transfer function in vacuum (0.9Pa) for $V_{\text {bias }}-V_{\text {ref }}=2 \mathrm{~V}$, $V_{\text {osc }}=-26 \mathrm{dBm}$ and $R_{\text {load }} M N / R_{\text {res }}=-68 \mathrm{~dB}$, resulting in $f_{\text {res }} \simeq 1.5 \mathrm{MHz}$ and $Q \simeq 8500 \ldots \ldots \ldots \ldots \ldots \ldots \ldots$

7 Experimental NEMS transfer function for different vacuum levels and $V_{\text {bias }}{ }^{-}$ $V_{\text {ref }}=1 \mathrm{~V}, V_{\text {osc }}=-20 \mathrm{dBm}, R_{\text {load }} M N / R_{\text {res }}=-76 \mathrm{~dB}$ and $f_{\text {res }} \simeq 1.5 \mathrm{MHz}$. . . . . 19

8 Experimental NEMS quality factor for different vacuum levels, under the same conditions of Figure 7. . . . . . . . . . . . . . . . . . 20

9 Experimental NEMS transfer function comparisons between vacuum (solid, $<5 \mathrm{~Pa}, V_{o s c}=-10 \mathrm{dBm}$ ) and air pressure (dashed, $V_{o s c}=0 \mathrm{dBm}$ ) for different $V_{\text {bias }}-V_{\text {ref }}$ levels. Due to limited frequency resolution, peak values are approximative. . . . . . . . . . . . . . . . . 21

10 Experimental (circles) and numerical fitting (solid) of the NEMS resonance frequency for different biasing levels $\left(2 \mathrm{~Pa}, V_{\text {osc }}=-20 \mathrm{dBm}\right) \ldots \ldots . \ldots 22$ 


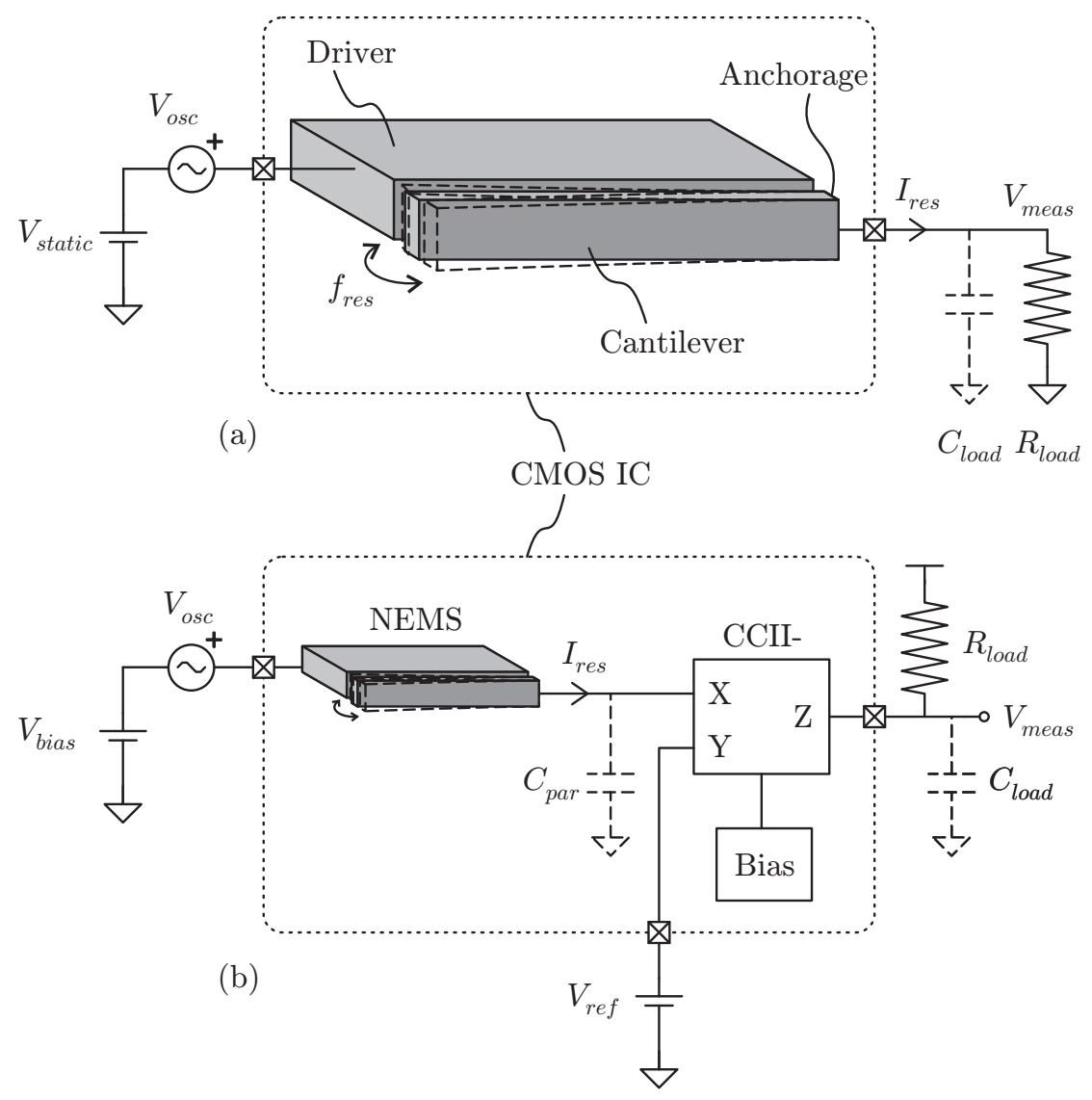

Figure 1: NEMS parts and ideal read out scheme (a), and proposed monolithic CMOS solution (b). 


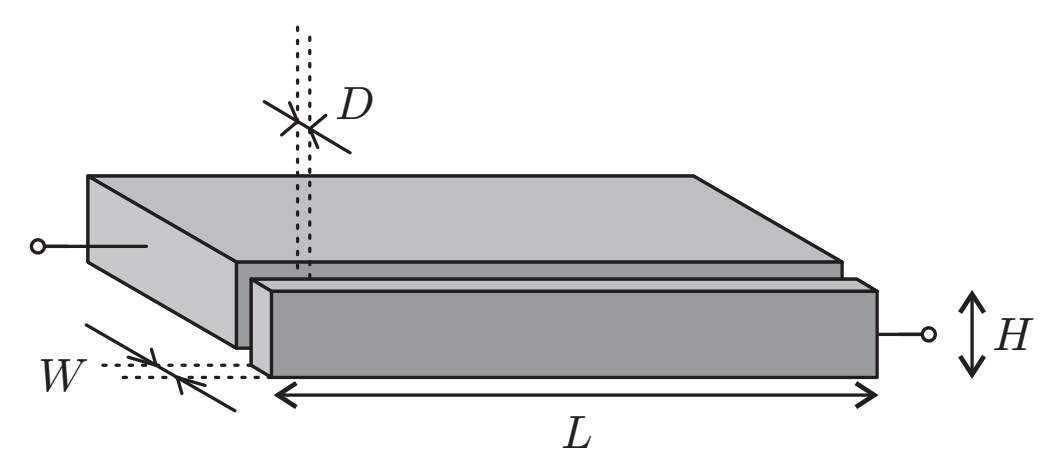

(a)

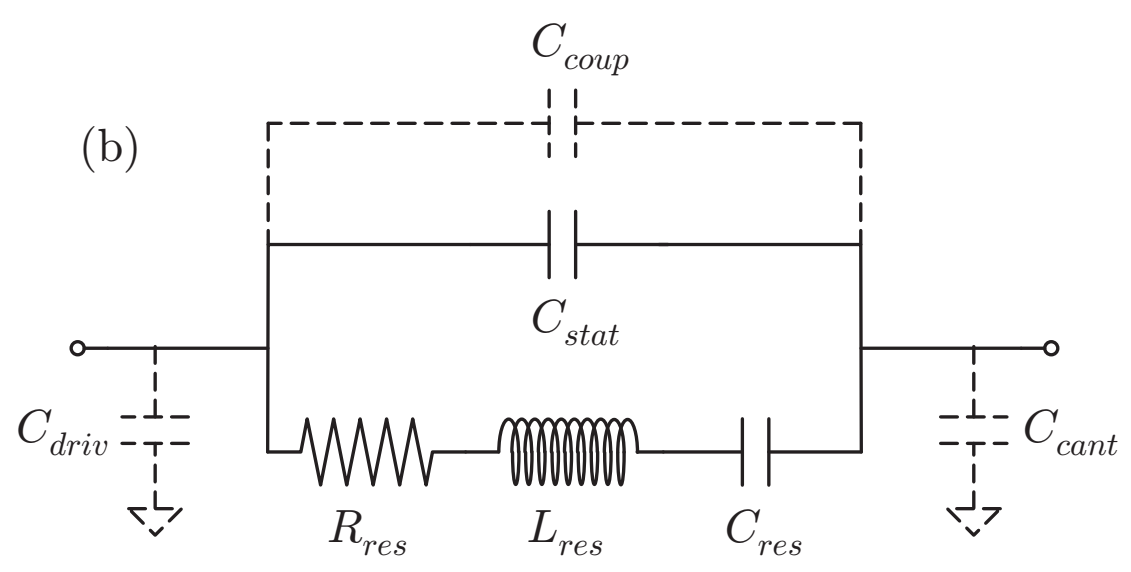

Figure 2: Mechanical (a) and electrical equivalent circuit model (b) of the NEMS resonator. Top drawing not in scale. 


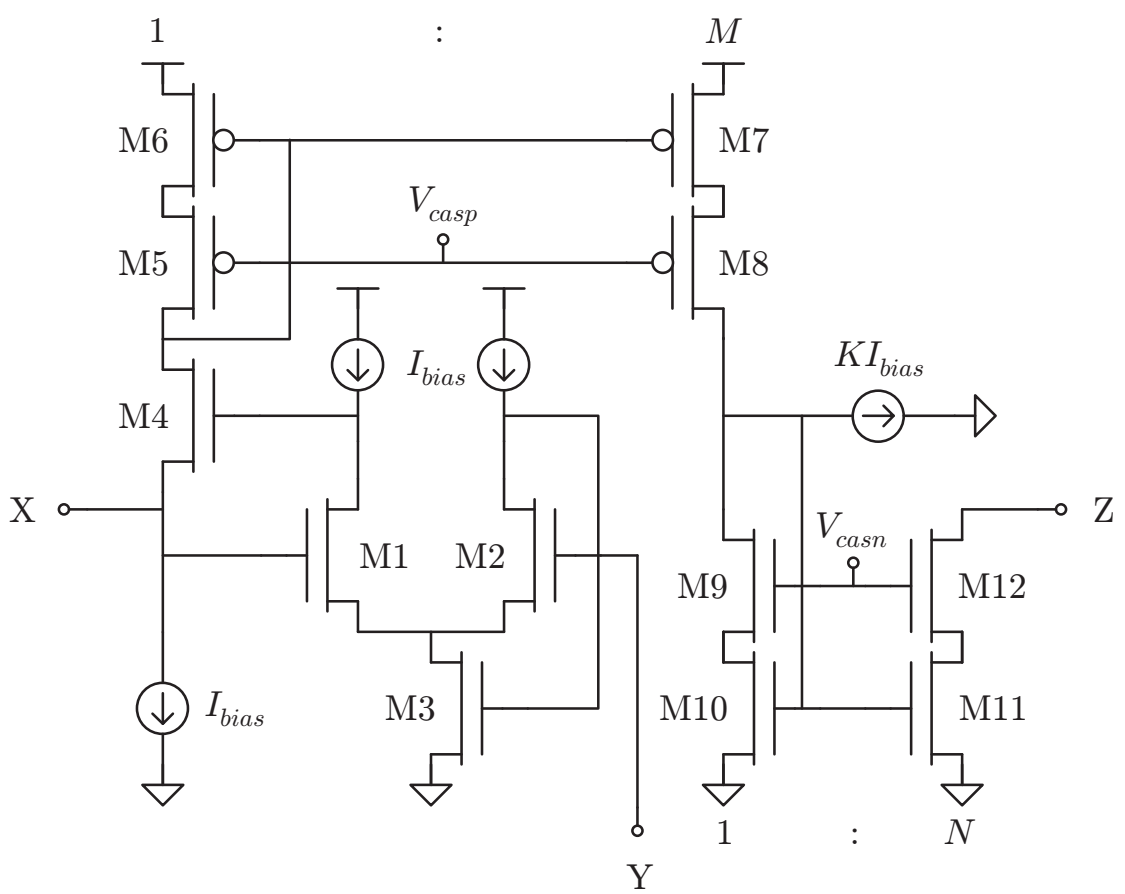

Figure 3: Simplified schematic of the CMOS CCII- circuit. 


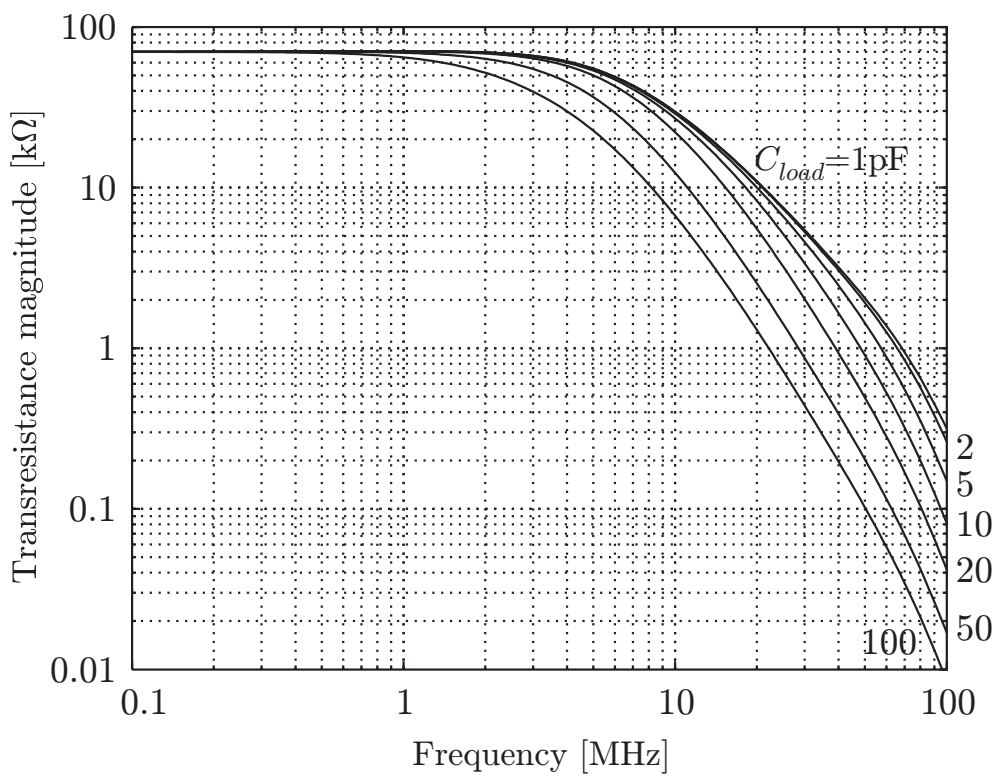

Figure 4: Simulated transfer function of the CCII- circuit of Figure 3 according to the design variables of Table 3 and for different load capacitance conditions. 


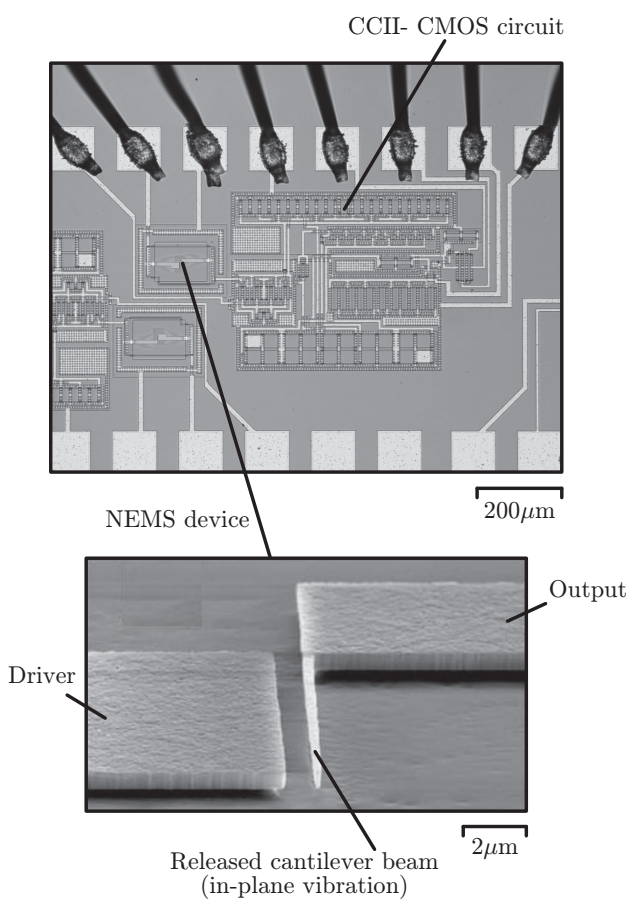

Figure 5: Microscope photography (top) of the mixed NEMS-CMOS circuit and SEM image (bottom) of the NEMS device. 

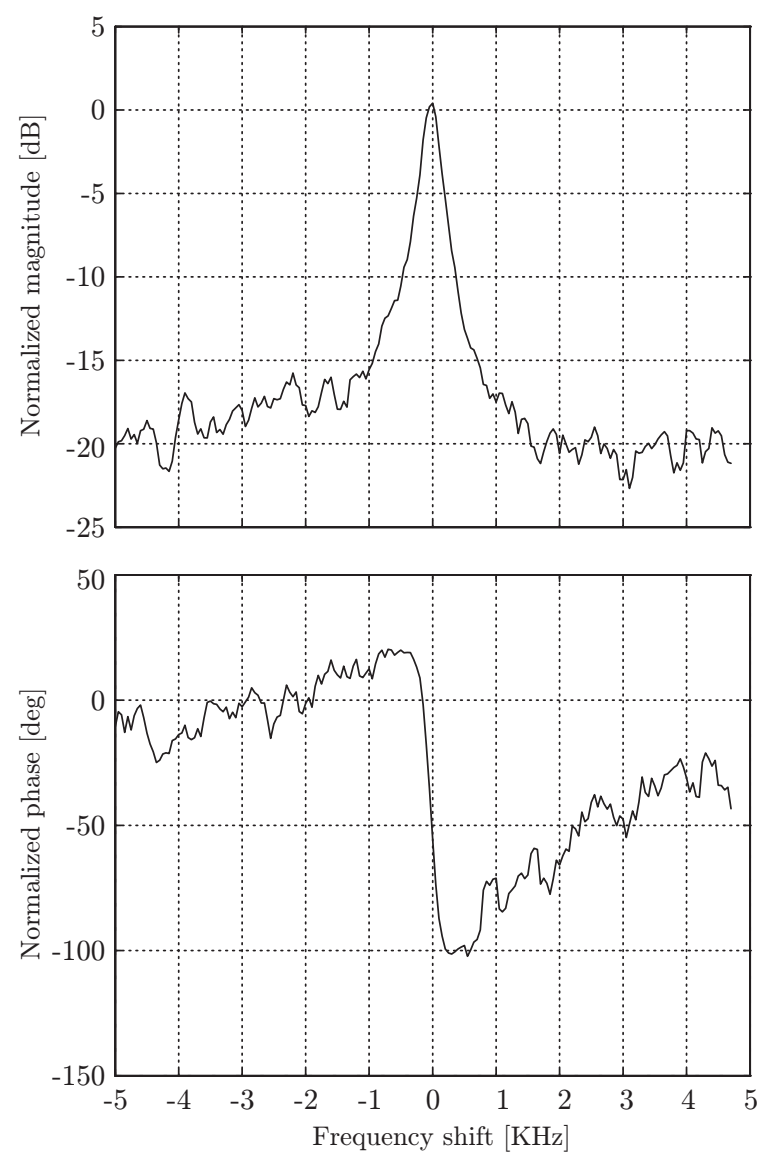

Figure 6: Experimental NEMS transfer function in vacuum $(0.9 \mathrm{~Pa})$ for $V_{\text {bias }}-V_{\text {ref }}=2 \mathrm{~V}$, $V_{\text {osc }}=-26 \mathrm{dBm}$ and $R_{\text {load }} M N / R_{\text {res }}=-68 \mathrm{~dB}$, resulting in $f_{\text {res }} \simeq 1.5 \mathrm{MHz}$ and $Q \simeq 8500$. 


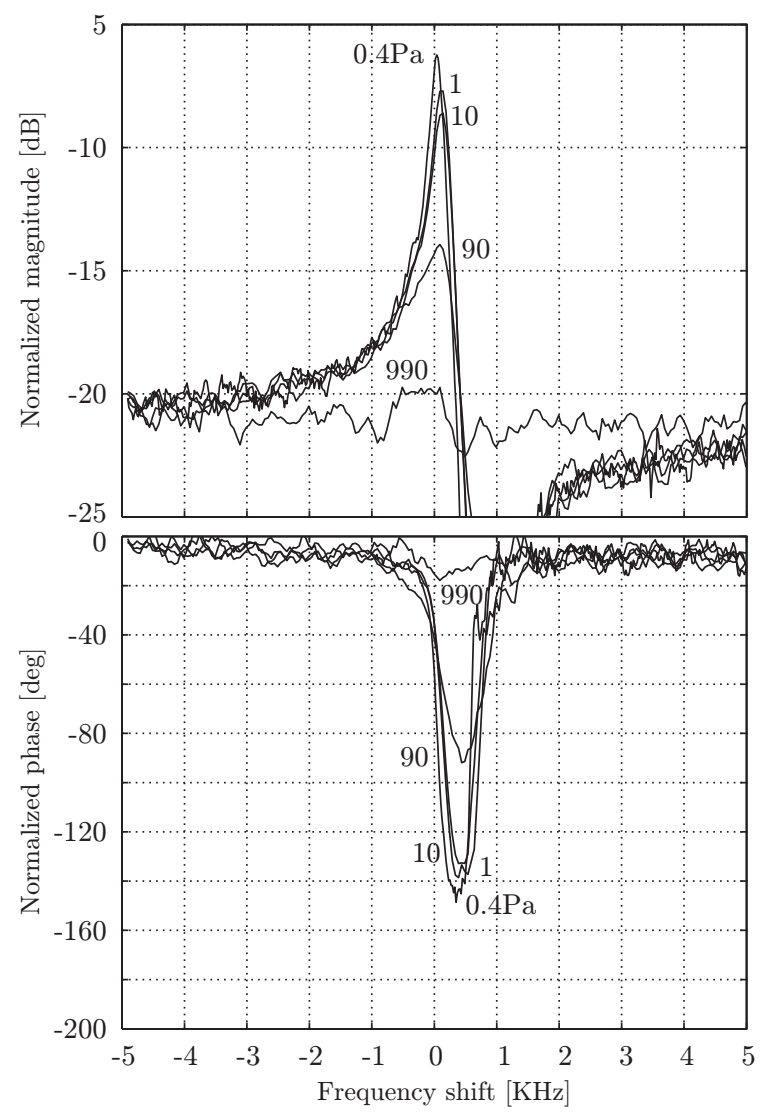

Figure 7: Experimental NEMS transfer function for different vacuum levels and $V_{\text {bias }}{ }^{-}$ $V_{\text {ref }}=1 \mathrm{~V}, V_{\text {osc }}=-20 \mathrm{dBm}, R_{\text {load }} M N / R_{\text {res }}=-76 \mathrm{~dB}$ and $f_{\text {res }} \simeq 1.5 \mathrm{MHz}$. 


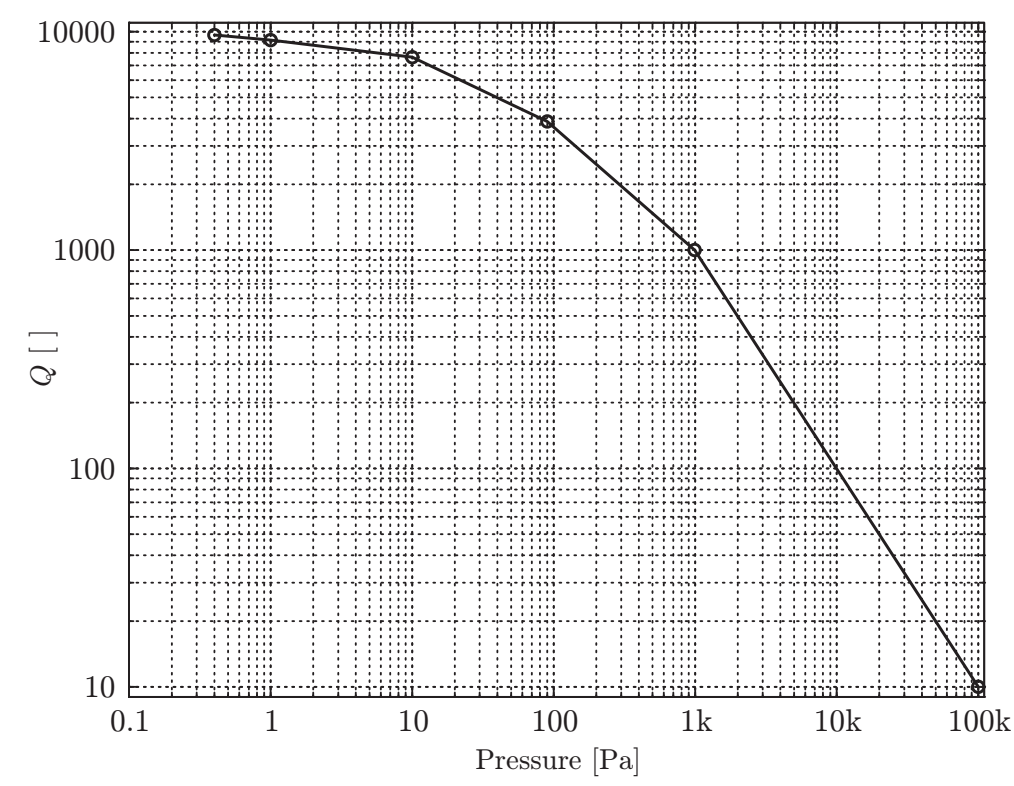

Figure 8: Experimental NEMS quality factor for different vacuum levels, under the same conditions of Figure 7. 

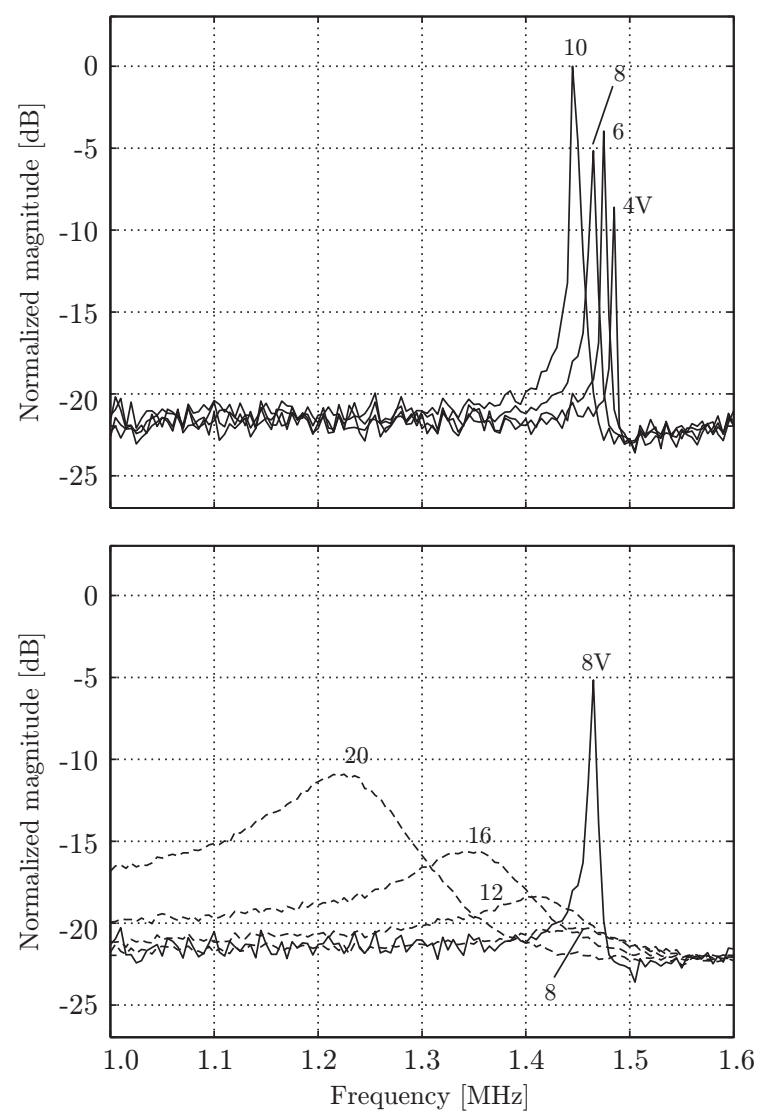

Figure 9: Experimental NEMS transfer function comparisons between vacuum (solid, $<5 \mathrm{~Pa}, V_{\text {osc }}=-10 \mathrm{dBm}$ ) and air pressure (dashed, $\left.V_{o s c}=0 \mathrm{dBm}\right)$ for different $V_{\text {bias }}-V_{\text {ref }}$ levels. Due to limited frequency resolution, peak values are approximative. 


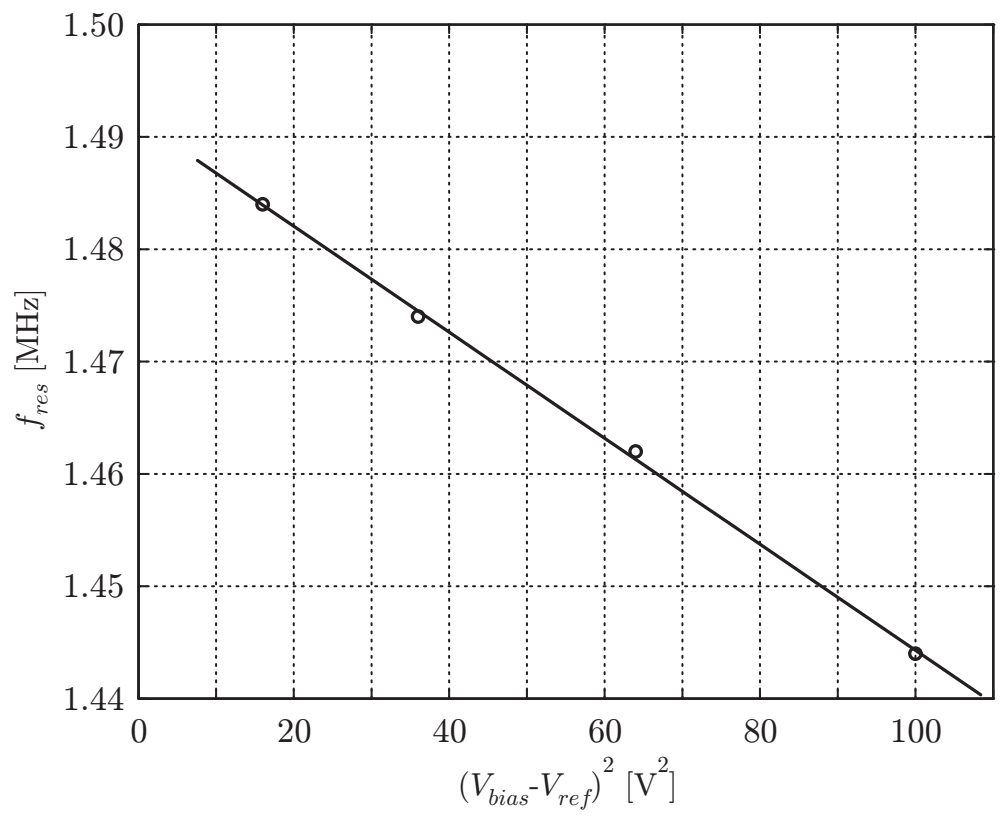

Figure 10: Experimental (circles) and numerical fitting (solid) of the NEMS resonance frequency for different biasing levels $\left(2 \mathrm{~Pa}, V_{\text {osc }}=-20 \mathrm{dBm}\right)$. 


\section{List of Tables}

1 Physical dimensions of the NEMS resonator of Figure 2. . . . . . . . . . . 24

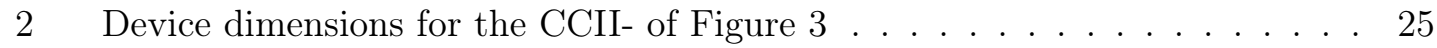

3 Overall performance of the read out circuit for $I_{b i a s}=8 \mu \mathrm{A}, M=N=10, K=9$, $R_{\text {load }}=700 \Omega$ and $C_{\text {load }}=30 \mathrm{pF} \ldots \ldots \ldots \ldots \ldots \ldots$

4 Equivalent RLC parameters extracted from the experimental results of Fig-

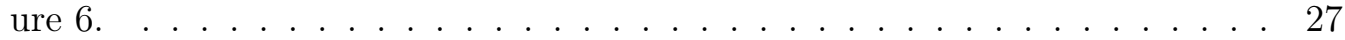




\begin{tabular}{lcl}
\hline Parameter & Value & Units \\
\hline$W$ & 260 & $\mathrm{~nm}$ \\
$L$ & 14.5 & $\mu \mathrm{m}$ \\
$H$ & 570 & $\mathrm{~nm}$ \\
$D$ & 820 & $\mathrm{~nm}$
\end{tabular}

Table 1: Physical dimensions of the NEMS resonator of Figure 2. 


\begin{tabular}{lr}
\hline Transistor & $\frac{W}{L}\left[\frac{\mu \mathrm{m}}{\mu \mathrm{m}}\right]$ \\
\hline M1-2 & $4 \times 30 / 5$ \\
M3 & $2 \times 15 / 10$ \\
M4 & $30 / 3$ \\
M5-6 & $10 / 5$ \\
M7-8 & $M \times 10 / 5$ \\
M9-10 & $50 / 5$ \\
M11-12 & $N \times 50 / 5$ \\
\hline
\end{tabular}

Table 2: Device dimensions for the CCII- of Figure 3 


\begin{tabular}{lcl}
\hline Parameter & Value & Units \\
\hline Transresistance & 70 & $\mathrm{~K} \Omega$ \\
Bandwidth & 4.5 & $\mathrm{MHz}$ \\
In-band input impedance & $<3$ & $\mathrm{~K} \Omega$ \\
In-band input current noise & 0.5 & $\mathrm{pA} / \sqrt{\mathrm{Hz}}$ \\
Supply voltage & 5 & $\mathrm{~V}$ \\
Current consumption & 185 & $\mu \mathrm{A}$ \\
\hline
\end{tabular}

Table 3: Overall performance of the read out circuit for $I_{\text {bias }}=8 \mu \mathrm{A}, M=N=10, K=9$, $R_{\text {load }}=700 \Omega$ and $C_{\text {load }}=30 \mathrm{pF}$. 


\begin{tabular}{lcl}
\hline Parameter & Value & Units \\
\hline$R_{\text {res }}$ & 84 & $\mathrm{M} \Omega$ \\
$L_{\text {res }}$ & 76 & $\mathrm{kH}$ \\
$C_{\text {res }}$ & 0.15 & $\mathrm{aF}$ \\
$C_{\text {stat }}+C_{\text {coup }}$ & 90 & $\mathrm{aF}$ \\
$C_{\text {cant }}$ & $<50$ & $\mathrm{fF}$ \\
\hline
\end{tabular}

Table 4: Equivalent RLC parameters extracted from the experimental results of Figure 6. 